\title{
ON BOUNDS FOR PARAMETERS IN $n$-NODED SOLUTIONS OF STURM-LIOUVILLE EQUATIONS*
}

\author{
J. K. L. MACDONALD
}

Generalized Sturm-Liouville differential equations $\dagger$ are of considerable importance, particularly in applied mathematics. Such equations have the form

$$
\left(\frac{d}{d x} p(x, \lambda) \frac{d}{d x}+q(x, \lambda)\right) y(x, \lambda)=0
$$

in which (in probably the most important cases) the independent variable $x$ ranges over a continuous finite or infinite real interval $(a, b)$ and in which values for the parameter $\lambda$ are implicitly determined by continuity and end conditions for $y$ and $(d y / d x)$. Fairly relaxed conditions are known $\ddagger$ to be sufficient to ensure the existence of a discrete set of $\lambda$ values $\lambda_{n},\left(n=n_{0}, n_{0}+1, \cdots\right)$, for which $\lambda_{n}$ corresponds to a unique solution $y=y_{n}$ with exactly $n$ nodes in $a<x<b$, $a$ and $b$ possibly being real singularities for the equation.

In this paper we shall assume that such conditions are satisfied and, in particular, that at least in the open interval $(a, b)$ the functions $p>0, y$, and $(d y / d x)$ are continuous, and that $(d p / d x)$ and $q$ are piecewise continuous functions of $x$. (We shall use the term piecewise continuity of $f(x)$ to imply continuity at least in an open interval concerned, except possibly for a finite number of points at which $f(x)$ is equal to one of the bounded unequal limits $f(x-0)$ and $f(x+0)$. Correspondingly we shall use $(d / d x)$ to denote left- or right-hand differentiation whichever is called for in a given expression.) We shall discuss methods for bounding $\lambda_{n}$ and

$$
\cot ^{-1}\left((p / q)^{1 / 2}(d / d x) \log (p q)^{1 / 4} y_{n}\right)
$$

by use of a phase angle $\phi$, and shall stress the accuracy of asymptotic forms $\S$ (as $n \rightarrow \infty$ ), a feature which has been little investigated in the past.

Let us consider an open interval $(\alpha, \beta)$ contained by $(a, b)$, and let

* Presented to the Society, September 7, 1937.

$\dagger$ See McCrea and Newing, Proceedings of the London Mathematical Society, (2), vol. 37 (1933), p. 520, for general discussion and references.

$\ddagger$ McCrea and Newing, loc. cit., p. 520 .

$\S$ See Langer, this Bulletin, vol. 40 (1934), p. 545, and Dunham, Physical Review, vol. 41 (1932). 
us introduce disposable continuous functions $r>0 *$ and $s$ with piecewise continuous $x$-derivatives $r^{\prime}$ and $s^{\prime}$. We define a dependent continuous function (phase angle) $\phi$ by the relation $\dagger$

$$
\cot \phi=s+\left(r p y^{\prime} / y\right) \text {. }
$$

Then we can eliminate $y^{\prime} / y$ between equations (1) and (2) to get the differential equation satisfied by $\phi$ :

$$
\begin{aligned}
\frac{d \phi}{d x}=\frac{1}{r p} & +\left(r q-\frac{1}{r p}+\frac{s^{2}}{r p}+\frac{r^{\prime} s}{r}-s^{\prime}\right) \sin ^{2} \phi \\
& -\left(\frac{r^{\prime}}{2 r}+\frac{s}{r p}\right) \sin 2 \phi .
\end{aligned}
$$

Our main concern is to choose the functions $r$ and $s$ so that equation (3) can be readily solved analytically or so that a comparison theorem can be used to bound $\phi$ closely between known forms. The field of choice is very wide and encourages individual ingenuity in special cases; so we shall concentrate on what appear to be the most useful general forms. Note that we can determine boundary values for $\phi$ by use of (2) and the asymptotic forms of $y^{\prime} / y$ near the boundaries, together with the property that $\phi$ passes through a multiple of $\pi$ only as $x$ passes through a nodal value for $y, \phi$ being an increasing function of $x$ near each node. Further we may note the useful suggestion that in generalf

$$
\int_{\alpha}^{\beta}(q / p)^{1 / 2} d x \sim n \pi \text { as } n \rightarrow \infty,
$$

where $n$ is the number of nodes in the interval $(\alpha, \beta)$ over which $q \geqq 0$ and over which the integral exists. In fact one of our main objects will be to determine the degree of accuracy of asymptotic relations like (4).

If we set $s=-p r^{\prime} / 2$, we rid (3) of the indefinite signed term $\sin 2 \phi$ :

$$
\frac{d \phi}{d x}=\frac{1}{p r}+\left(r q-\frac{1}{p r}-\frac{p\left(r^{\prime}\right)^{2}}{4 r}+\frac{\left(p r^{\prime}\right)^{\prime}}{2}\right) \sin ^{2} \phi .
$$

* This condition may be relaxed to allow $r \rightarrow 0, r \rightarrow \infty$, or $r$ to be only piecewise continuous within $(\alpha, \beta)$, but then it may be difficult to determine the possible discontinuities in $\phi$.

$\dagger$ Prüfer, Mathematische Annalen, vol. 95 (1926), pp. 499-518, was the first to make general use of a polar angle. His form corresponds to setting $s=0$ and $r=1$ in our general expression.

† Langer, loc. cit., Dunham, loc. cit. 
In particular, we can take $(\alpha, \beta)$ as an open interval over which $p q>0^{*}$ and over which $(p q)$ and $(p q)^{\prime}$ are continuous and $(p q)^{\prime \prime}$ piecewise continuous. Then we can set $r=(p q)^{-1 / 2}$ and get, as a result, the form

$$
\frac{d \phi}{d x}=\left(\frac{q}{p}\right)^{1 / 2}\left(1+F \sin ^{2} \phi\right),
$$

with

$$
\begin{aligned}
\cot \phi & =\left(\frac{(p q)^{\prime}}{4 p q}+\frac{y^{\prime}}{y}\right)\left(\frac{p}{q}\right)^{1 / 2}, \\
F & =\frac{5}{16 p q^{3}}\left(\left(\frac{d}{d x} p q\right)^{2}-\frac{4}{5}\left(q \frac{d}{d x} p \frac{d}{d x} p q\right)\right) .
\end{aligned}
$$

We have much reason to regard equation (6) as our most simple and powerful form for discussing many types of examples, and in particular for determining the accuracy of the relation (4). For such purposes we can use the following among the many inequalities derivable directly from (6) or through a comparison theorem. $\dagger$ We take $(c, d)$ to be any appropriate interval contained by $(\alpha, \beta)$, with $\phi_{c}$ and $\phi_{d}$ the values for $\phi$ at $x=c$ and $x=d$, respectively. Then we obtain the relations

$$
\int_{c}^{d}\left(\frac{q}{p}\right)^{1 / 2} d x \leqq \phi_{d}-\phi_{c} \quad\left(\int_{c}^{d}\left(\frac{q}{p}\right)^{1 / 2} d x \geqq \phi_{d}-\phi_{c}\right)
$$

if $0 \leqq F(0 \geqq F)$ over the interval $(c, d)$, which is a special case of the stronger form

$$
\begin{gathered}
I \leqq \int_{c}^{d}\left(\frac{q}{p}\right)^{1 / 2} d x \leqq I-\int_{c}^{d}\left(\frac{q}{p}\right)^{1 / 2}\left(\frac{F-k}{1+k}\right) d x \\
\left(I \geqq \int_{c}^{d}\left(\frac{q}{p}\right)^{1 / 2} d x \geqq I-\int_{c}^{d}\left(\frac{q}{p}\right)^{1 / 2}\left(\frac{F-k}{1+k}\right) d x\right)
\end{gathered}
$$

if $k>-1$ is an upper (a lower) bound for $F$ over $(c, d)$, where

$$
I=\int_{\phi_{c}}^{\phi_{d}} \frac{d \phi}{1+k \sin ^{2} \phi}=\left.\frac{1}{2(1+k)^{1 / 2}} \sin ^{-1} \frac{1-(2+k) \sin ^{2} \phi}{1+k \sin ^{2} \phi}\right|_{\phi_{c}} ^{\phi_{d}}
$$

(evaluated as an increasing function of $\phi_{d}$ ). Furthermore

* See the first footnote on p. 165.

$\dagger$ See, for example, Kamke's Differentialgleichungen, pp. 82-86. We use the generalized form with $\leqq$ replacing $<$, and with $\phi(\xi) \leqq \psi(\xi)$ in Kamke's theorem on p. 82 . 


$$
\begin{gathered}
\int_{c}^{d}\left(\frac{q}{p}\right)^{1 / 2}(1+F) d x \leqq \phi_{d}-\phi_{c} \\
\left(\int_{c}^{d}\left(\frac{q}{p}\right)^{1 / 2}(1+F) d x \geqq \phi_{d}-\phi_{c}\right)
\end{gathered}
$$

if $F \leqq 0(F \geqq 0)$ over $(c, d)$, which is a special case of the general form

$$
\begin{gathered}
\int_{c}^{d}\left(\frac{q}{p}\right)^{1 / 2}\left(1+\gamma^{2} F\right) d x \leqq \phi_{d}-\phi_{c} \\
\left(\int_{c}^{d}\left(\frac{q}{p}\right)^{1 / 2}\left(1+\gamma^{2} F\right) d x \geqq \phi_{d}-\phi_{c}\right)
\end{gathered}
$$

if $\gamma^{2} F \leqq F \sin ^{2} \phi\left(\gamma^{2} F \geqq F \sin ^{2} \phi\right)$ over $(c, d)$, where $\gamma$ is some prescribed function usually suggested by the asymptotic form of $\sin ^{2} \phi$ near the end points $c$ and $d$. We can usually find suitable asymptotic forms by use of $\cot \phi$ in (6) and the Riccati form for (1), namely,

$$
\frac{d}{d x} p\left(\frac{y^{\prime}}{y}\right)+p\left(\frac{y^{\prime}}{y}\right)^{2}+q=0 \text {. }
$$

Let us illustrate the use of the above inequalities. For examples in which $(q / p)^{1 / 2}$ and $(q / p)^{1 / 2} F$ are integrable over the interval between the end points at which the boundary conditions are given, we may readily bound $\lambda_{n}$, for instance, as in the following example. We consider the nonsingular equation $\left(d^{2} y / d x^{2}\right)+\lambda^{2} x^{2} y=0$ subject to $y=0$ at $x=1$ and at $x=2$. Here in (6) we have

$$
F=\frac{3}{4 \lambda^{2} x^{4}}, \quad \frac{3}{4 \lambda^{2}} \geqq F \geqq \frac{3}{64 \lambda^{2}},
$$

and $y^{\prime} / y \sim 1 /(x-1)+1 /(x-2)$ near $x=1,2$ (by (9) and the boundary conditions). Therefore, by $\cot \phi$ in (6) we have $\phi=0$ at $x=1$ and $\phi=(n+1) \pi$ at $x=2$, when $n$ is the number of nodes within $(1,2)$. We therefore have the following bounding relations for $\lambda=\lambda_{n}$ : From (7a),

$$
\frac{3}{2} \lambda_{n} \leqq(n+1) \pi
$$

From the left inequality* in (7b),

$$
(n+1) \pi\left(1+\frac{3}{4 \lambda^{2}}\right)^{-1 / 2} \leqq \frac{3 \lambda}{2} \leqq(n+1) \pi\left(1+\frac{3}{64 \lambda^{2}}\right)^{-1 / 2} ;
$$

* The right-hand side of $(7 \mathrm{~b})$ yields very close but more complicated bounds. 
whence, for example, in the case of $n-1$ nodes within $(1,2)$,

$$
\frac{2}{3}\left(n^{2} \pi^{2}-\frac{27}{16}\right)^{1 / 2} \leqq \lambda_{n-1} \leqq \frac{2}{3}\left(n^{2} \pi^{2}-\frac{27}{256}\right)^{1 / 2} \text {. }
$$

From (8a),

$$
\frac{3 \lambda_{n-1}}{2}+\frac{9}{32 \lambda_{n-1}} \geqq n \pi,
$$

which gives us a larger lower bound for $\lambda_{n-1}$. With little more trouble we could find even closer bounds for $\lambda_{n-1}$; for example, by dividing the interval $(1,2)$ into subintervals $(c, d)$.

In examples for which there are points near which at least one of the expressions $(q / p)^{1 / 2}$ and $(q / p)^{1 / 2} F$ is not integrable we must, in general, use a comparison theorem to supplement the inequalities (7) and (8) in order to obtain both upper and lower bounds. Let us consider an example which involves singular end points and is not yet solved in closed form: $p=1-x^{2}, q=\lambda^{2} x$, subject to the condition $y$ bounded throughout the interval $-1<x<1$. In this case we have the intervals $(a, b) \equiv(-1,1),(\alpha, \beta) \equiv(0,1)$, and, using $(2)$ and the appropriate exponents at $x= \pm 1$, we obtain $\phi=0$ at $x=0^{*}$ and $\phi=(n+1) \pi$ at $x=1$. We introduce the constants

$$
B=\int_{0}^{1}\left(\frac{x}{1-x^{2}}\right)^{1 / 2} d x=\left(\frac{2}{\pi}\right)^{1 / 2} \Gamma^{2}\left(\frac{3}{4}\right) \doteq \frac{2}{3} \pi^{1 / 2}, \quad C \doteq 1.71,
$$

where $C$ is the minimum value (in the interval $0<x<1$ ) of

$$
\lambda^{2} F=\left(3 x^{2}-5\right)\left(x^{2}+1\right) / 16 x^{3}\left(x^{2}-1\right) \text { at } x^{2}=(8 / 3)^{1 / 2}-1 .
$$

Therefore from the left inequality in (7b) we get the bounding relation

$$
\lambda_{n-1} \leqq\left((n \pi / B)^{2}-C\right)^{1 / 2}<n \pi / B \cdot \dagger
$$

Before considering such examples further let us note the following useful comparison $\ddagger$ forms for use with (6):

$$
(d \theta / d x)=(q / p)^{1 / 2}\left(1+F \theta^{2}\right)
$$

* In this case $p y^{\prime} / y$ has a finite nonzero value at $z=0$ if $y$ is bounded at $z=-1$, because of the general identity for (1); namely $\left.p y y^{\prime}\right|_{x_{1}} ^{x_{2}}=\int_{x_{1}}^{x_{2}}\left(p\left(y^{\prime}\right)^{2}-q y^{2}\right) d x$ (whence $p y y^{\prime}=0$ at most once in an interval where $q \leqq 0$ but $y^{\prime} \not \equiv 0$ if $q \neq 0$ ).

$\dagger$ In Legendre's equation $p=1-x^{2}, q=\lambda^{2}$, and $(a, b) \equiv(\alpha, \beta) \equiv(-1,1)$. Here (7b) yields the inequality $\lambda_{n}{ }^{2}<(n+1)^{2}-1 / 2$ for any solution with $n$ nodes in $(-1,1)$.

$\ddagger$ See the second footnote on p. 166. 
which is satisfied by $\theta=4 q(p q)^{1 / 2} /(p q)^{\prime}$; and

$$
(d \psi / d x)=(q / p)^{1 / 2}(1+F f(x) g(\psi-t))
$$

which is satisfied by the form $\psi$ given by the equation

$$
\int \frac{d(\psi-t)}{g(\psi-t)}=\int \frac{f(x) d(\psi-t)}{\theta^{2}}=\int f(x)(q / p)^{1 / 2} F d x .
$$

We have used the following symbols in the above equations:

$$
\theta=4 q(p q)^{1 / 2} /(p q)^{\prime}, \quad t=\int(q / p)^{1 / 2} d x,
$$

with $f(x)$ and $g(\psi-t)$ disposable functions. In particular, we can set $f(x) g(\psi-t)=h \sin ^{2} j(\psi-t)$ in (12b) and (12c) ( $h$ and $j$ any constants) and obtain

$$
\begin{aligned}
(d \psi / d x) & =(q / p)^{1 / 2}\left(1+F h \sin ^{2}(\psi-t)\right), \\
\psi & =t-j^{-1} \cot ^{-1} \int j h(q / p)^{1 / 2} F d x .
\end{aligned}
$$

Using the forms $\phi$ of (6) and $\psi$ of (12) in the comparison theorem* we have the following: If in some interval $x_{1}<x<x_{2}$ or $x_{1}>x>x_{2}$ (over which $\phi$ and $\psi$ are bounded in any given example) it can be shown that $\phi \geqq \psi(\phi \leqq \psi)$ near $x=x_{1}$, then the same inequality will continue to hold throughout the interval if (correspondingly)

$$
\begin{aligned}
\left(x_{2}-x_{1}\right) F \sin ^{2} \psi & \geqq\left(x_{2}-x_{1}\right) F f(x) g(\psi-t) \\
\left(\left(x_{2}-x_{1}\right) F \sin ^{2} \psi\right. & \left.\leqq\left(x_{2}-x_{1}\right) F f(x) g(\psi-t)\right)
\end{aligned}
$$

throughout the interval.

For examples in which $(q / p)$ or $F$ lead to unbounded integrals (or to poor bounds) in forms like (7) and (8), if $c \rightarrow \alpha$ and (or) $d \rightarrow \beta$, we can take $(c, d)$ to be interior to $(\alpha, \beta)$ and use (13) to fill in between $\alpha$ and $c$ and $d$ and $\beta$. We may thus find bounds for $\phi_{c}$ and $\phi_{d}$ in forms like (7b), or for the right-hand side of an equation such as the following (corresponding to $(7 \mathrm{~b}))$ :

$$
\begin{aligned}
\int_{\phi_{\alpha}}^{\phi_{\beta}} \frac{d \phi}{1+k \sin ^{2} \phi}-\int_{\alpha}^{\beta}\left(\frac{q}{p}\right)^{1 / 2} d x & \\
& =\int_{\alpha}^{\beta}\left(\frac{q}{p}\right)^{1 / 2} \frac{(F-k) \sin ^{2} \phi}{1+k \sin ^{2} \phi} d x .
\end{aligned}
$$

* See the second footnote on p. 166. 
For example, we may bound the right-hand side of (14) (usually after preliminary use of symmetry, integration by parts, and other simple manipulations) by breaking the interval of integration into $(\alpha, c)$, $(c, d)$, and $(d, \beta)$, and replacing $\phi$ in the first and third intervals by appropriate comparison $\psi$ 's and replacing $\sin ^{2} \phi$ in the second interval by 0 or 1 as in (7b). We should choose $(c, d)$ so as to give the smallest (absolute value) bound for the right-hand side of (14). Using the latter method with, for example, $\psi=\theta>\phi$ near $x=-1$ in the case of Legendre's polynomials, ${ }^{*}$ we can readily find such very good bounds as

$$
\left(n+\frac{1}{2}-\frac{1}{\pi^{2}}\right)^{2}-\frac{1}{2}<\lambda_{n}^{2}\left(=\left(n+\frac{1}{2}\right)^{2}-\frac{1}{4}\right) .
$$

In the case mentioned before, with $p=\left(1-x^{2}\right)$ and $q=\lambda^{2} x$, we can proceed in a very similar manner, except that we must use the Riccati equation (9) or its equivalent in order to get a suitable approximate value for $\phi$ at $x=0 \dagger$ in terms of the value of $y^{\prime} / y$ at $x=-1$. We can quite easily get upper and lower bounds for $\lambda_{n}$ isolating it from its neighbors $\lambda_{n-1}$ and $\lambda_{n+1}$. Such isolating of the possible ranges for the parameter values is very useful if, as in this case, we can determine the values by means of a continued fraction or other transcendental equation.

Thus far we have considered the form (5) only under the special substitution $r=(p q)^{-1 / 2}$. We can, in general, formally simplify the bounding process by using a substitution in (5) such as

$$
r=(p q)^{-1 / 2} \tanh k(p q)^{1 / 2}, \quad k>0 \text { any constant, }
$$

which has the property of differing slightly from $(p q)^{-1 / 2}$ where $k(p q)^{1 / 2}$ is large, but of not introducing unboundedness in the coefficient of $(p r)^{-1} \sin ^{2} \phi$ near end points. Under such circumstances if $\int d x / p r$ is bounded, we can use forms corresponding to (7) and (8) for obtaining the desired bounds. Using this procedure we may encounter more complicated integrals which may require series expansion or some such device in order to evaluate them. We may frequently find it profitable to use this method to bound $\left.\phi\right|_{c} ^{a}$ in (7) or (8) over an interval $(c, d)$ in which $F$ is bounded, instead of using comparison forms such as in (14).

In cases where the function $(p q)$ violates the conditions required for (6) and (15), we must use other substitutions for $r$, being guided in

* See the second footnote on p. 168.

† See the first footnote on p. 168. 
general, by the desirability of having $r \sim(p q)^{-1 / 2}$ in regions over which the latter varies little about a small value, and of having $(\alpha, \beta) \equiv(a, b)$ in (3). Here we should note the following general form for (5). If in (5) we set $r=\rho^{2}>0$ in $\alpha<x<\beta$, then

$$
\frac{d \phi}{d x}=\frac{1}{p \rho^{2}}+\sin ^{2} \phi \rho\left(\frac{d}{d x} p \frac{d}{d x}+q-\frac{1}{p \rho^{3}}\right) \rho .
$$

Further if the function $\sigma=\sigma(x)$ satisfies an equation

$$
\left(\frac{d}{d x} p \frac{d}{d x}+q-\delta\right) \sigma=0,
$$

where $\sigma$ and $\sigma^{\prime}$ are continuous and $\delta$ is piecewise continuous over $\alpha<x<\beta$, then if we define continuous functions $\rho>0$ and $\psi$ by the relation

$$
\rho e^{i \psi}=\sigma\left(1+i \int d x / p \sigma^{2}\right)
$$

we have

$$
\frac{d \phi}{d x}=\frac{1}{p \rho^{2}}+\delta \rho^{2} \sin ^{2} \phi=\frac{d \psi}{d x}+\delta \rho^{2} \sin ^{2} \phi
$$

with $y=\rho(\sin \phi) \exp \left\{-\int \delta \rho^{2} \sin \phi \cos \phi d x\right\}$.

In using (16) or (17) we ordinarily wish to choose $\rho$ (or $\sigma$ ) so that the coefficient of $\sin ^{2} \phi$ is one-signed over $(\alpha, \beta)$, or so that bounding integrals analogous to those in (7) and (8) assume sufficiently small values.

Among substitutions other than $2 s=-p r^{\prime}$ (which led to (5)) we note the following:

$$
r=(p q)^{-1 / 2}, \quad s=0 ;(d \phi / d x)=(q / p)^{1 / 2}+(\sin 2 \phi)(p q)^{\prime} /(4 p q)^{*}
$$

which is useful if, for example, $(p q)^{\prime} /(4 p q)$ is small compared to the continuous function $(q / p)^{1 / 2}$, even if the former is only piecewise continuous throughout $(\alpha, \beta)$. Special examples may suggest simple substitutions to give us the desired bounds in similar examples.

\section{Cornell University}

* A possible comparison form here is $(d \psi / d x)=(q / p)^{1 / 2}+f(x) g(\psi-t)$ (similar to $(12 b))$. 